\title{
Radon-therapy in ankylosing spondylitis reduces auto-antibody titers
}

\author{
Angelika Moder ${ }^{1,2}$, Clemens Hufnagl ${ }^{1,2}$, Martin Jakab ${ }^{1}$, Wolfgang Hitzl ${ }^{3}$, Markus Ritter ${ }^{1,2}$ \\ ${ }^{1}$ Institute of Physiology and Pathophysiology, Paracelsus Medical University, Salzburg, Austria; \\ ${ }^{2}$ Gastein Research Institute, Paracelsus Medical University, Bad Gastein, Austria; \\ ${ }^{3}$ Research Office, Biostatistics, Paracelsus Medical University, Salzburg, Austria. \\ Email: angelika.moder@pmu.ac.at
}

Received 2 August 2011; revised 15 September 2011; accepted 27 September 2011.

\begin{abstract}
Combined low-dose radon/hyperthermia therapy has been reported to improve pain and mobility in patients with rheumatic disorders. The key feature of inflammatory rheumatic diseases is the induction of autoimmune processes via the production of autoantibodies. As reducing the autoantibody level through administration of biologicals is described to correlate with delay of disease progression, we investigated the impact of combined low-dose radon/ hyperthermia therapy on the serum levels of antibodies directed against cyclic citrullinated peptides in patients with ankylosing spondylitis. We found that levels of circulating anti-CCP antibodies is significantly reduced immediately after a 3 weeks of radon therapy regimen.
\end{abstract}

Keywords: Radon; CCP; Autoantibodies; Ankylosing Spondylitis

\section{INTRODUCTION}

Ankylosing spondylitis (AS) is a chronic inflammatory disorder, which is mainly characterized by tissue destruction in axial joints and bilateral sacroiliitis. Sometimes, peripheral joints and extra-articular organs such as lung and heart valves are also affected. Autoimmunity - the key feature of rheumatoid disorders including AS-manifests itself via production of antibodies against self" antigens. Among several environmental and genetic factors that seem to predispose for rheumatoid arthritis (RA), formation of neo-antigens via altered post-translational modifications is believed to be strongly associated with the onset of autoimmunity and, subsequently, the pathogenesis of rheumatoid disorders. In fact, genetic polymorphisms of peptidyl-arginine-deiminase type IV (PAD IV), the enzyme that mediates citrullination of proteins, has been associated with the susceptibility to RA [1]. Antibodies against cyclic citrullinated proteins (anti-CCP) target multiple citrullinated proteins and are highly specific for RA [2-4]. Thus, anti-CCPs have become a useful diagnostic marker and a predictive tool for the progression of RA $[5,6]$.

B cell depletion and, thus, reducing circulating autoantibodies, has proven successful in patients with RA [7] and, similarly, medication with TNF- $\alpha$ blocking agents reduced disease severity and auto-antibody levels [8-11].

Combined low-dose radon/hyperthermia therapy (LDRnHT) has been reported to improve mobility and reduce pain in patients with AS [12]. However, the underlying mechanisms are still unclear. In a pilot study on 33 patients suffering from AS, we investigated the effect of LDRnHT on the level of circulating anti-CCPs.

\section{PATIENTS AND METHODS}

The study was designed as pilot study and was approved by the ethics committee of the state of Salzburg, Austria. Written informed consent was obtained from all patients. Inclusion criteria were age: age 20 - 75 years, diagnosed spondylitis ankylosans based on the modified New York Criteria for the Diagnosis of ankylosing spondylitis, physician prescribed LDRnHT and previously given written consent. Exclusion criteria were: therapy with either glucocorticoids, bisphosphonates, parathyroid hormone, vitamin-D or strontium renalate four weeks prior or during the study, treatment with TNF- $\alpha$ blockers or other biologicals eight weeks prior or during the study as well as hormone replacement therapy in post-menopausal women. A total of 33 patients ( 8 women, 25 men) median aged 53 (ranging from 21 - 74) years were enrolled without performing new radiographic examinations before enrolment. The median duration of disease was 28 years (ranging from 10 - 58). 14 patients received NSAIDs. LDRnHT was performed in the thermal tunnels of Boeckstein-Bad Gastein with a total adminis- 
tra- tion of ten treatments during three weeks. Each treatment was 90 minutes at $37^{\circ} \mathrm{C}-40.5^{\circ} \mathrm{C}$ ambient temperature, $70 \%-95 \%$ air humidity and radon $(222 \mathrm{Rn})$ activity of $\sim 4.5 \mathrm{nCi} / \mathrm{L}$. For ethical reasons, which would have been either refusal of a physician prescribed therapy or an unreasonable radiation exposure of persons lacking a therapeutic indication, no control group was enrolled in this pilot study.

\subsection{Blood Serum Preparation}

Forearm venous blood samples were drawn immediately before the first and immediately after the last treatment. Blood was collected in monovettes (Becton Dickinson, NJ, USA), incubated for $15 \mathrm{~min}$ at room temperature and centrifuged for $15 \mathrm{~min}$. Serum was stored in aliquots at $-20^{\circ} \mathrm{C}$ prior to analysis.

\subsection{Enzyme-Linked Immunosorbent Assay (ELISA)}

ELISAs were performed according to the manufacturers instructions. Anti-CCP titer determination kit was purchased from Euroimmun (EuroimmunAG Lübeck, Germany). Values above upper limit of quantification were set as equal to maximum value of the standard-curve within linear range.

\subsection{Statistical Analysis}

Data were evaluated for normal distribution prior to statistical analysis. Data are expressed as mean \pm SEM. Statistical analysis included descriptive statistic such as means, upper and lower quartile and standard error to the mean (SEM). Data were evaluated for normal distribution prior to statistical analysis using KolmogoroffSmirnov and Lilifors test. Statistical analysis was performed using a Wilcoxon signed rank test as a non-parametric method. A p-value less than 5\% was considered to indicate a statistically significant effect. All analyses were done using StatXact 6.0 (Cytel Software Corporation, Cambridge MA 2002).

\section{RESULTS}

\section{Decreased Titers of Circulating Anti-CCPs}

Mean titers of anti-CCPs in peripheral blood signifycantly decreased by $12.2 \pm 2.4 \%(\mathrm{p}<0.0001)$ from $100.3 \pm 5.6$ to $85.4 \pm 4.0 \mathrm{pg} / \mathrm{mL}$. Median decreased from 84.8 to $69.0 \mathrm{RU} / \mathrm{mL}$ (Figure 1). Individual concentrations ranged from $71.8 \mathrm{RU} / \mathrm{mL}$ to $200 \mathrm{RU} / \mathrm{mL}$.

\section{DISCUSSION}

The clinical significance in terms of predictive as well as diagnostic value of autoantibodies, particularly those directed against cyclic citrullinated proteins, has been well documented.

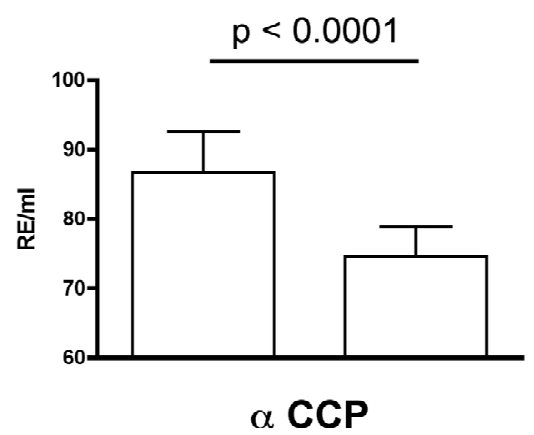

Figure 1. Titers of anti-CCP antibodies before (left) and after (right) LDRnHT (median \pm SEM).

Presence of anti-CCPs before onset as well as in established RA is associated with more severe radiological damage [13] and thus disease severity [14]. In a pilot study on 33 patients, we could clearly demonstrate a significant decrease of anti-CCPs in AS patients after receiving LDRnHT. Due to the pilot nature of our study we could not yet demonstrate the sustainability of the observed effect. Nevertheless our findings are encouraging since they are in agreement with those obtained in course of other treatment modalities for rheumatic disorders. Combating the chronic inflammation in RA with TNF- $\alpha$ blocking agents showed a significant decrease of anti-CCP levels in several studies [9-11]. Although, there are conflicting results derived from a few other studies, the majority observed a decrease in serum anti-CCP titer. Notably, Alessandri et al. reported a significant decrease in serum anti-CCPs in patients who benefitted from clinical improvement in contrast to those, who did not [8]. This suggests a causal correlation between alleviation in disease severity and a decrease in autoantibody levels.

From a pathophysiological point of view, the selfmaintenance of rheumatoid synovitis can be, at least partially, explained by continuous activation of antigenpresenting cells or phagocytes via antigen/autoantibody immune-complexes. Both, the predominant anti-CCP isotype IgG1 as well as the less frequently found IgG4 [2] can trigger the high affinity activating receptor Fc $\gamma$ RIIIa found on phagocytes. In addition, IgG1 is a potent activator of the complement cascade leading to destruction of target cells and thereby further contribute to the inflammatory processes. Taken together, reducing the autoantibody level may dramatically interfere with several inflammatory pathways that probably synergize with others and cause tissue destruction, pain and progression of disease.

Our finding that LDRnHT leads to a reduction of anti-CCP levels in patients with AS further substantiates the already reported beneficial effects of this treatment [12] and will prompt us to verify these data in a placebo- 
controlled study and to further investigate the underlying biological mechanisms that are still poorly understood.

\section{REFERENCES}

[1] Van Der Helm-Van Mil, A.H., Wesoly, J.Z. and Huizinga, T.W. (2005) Understanding the genetic contribution to rheumatoid arthritis. Current Opinion in Rheumatology, 17, 299-304. doi:10.1097/01.bor.0000160780.13012.be

[2] Chapuy-Regaud, S., et al. (2005) IgG subclass distribution of the rheumatoid arthritis-specific autoantibodies to citrullinated fibrin. Clinical and Experimental Immunology, 139, 542-550. doi:10.1111/j.1365-2249.2004.02708.x

[3] Schellekens, G.A., et al. (1998) Citrulline is an essential constituent of antigenic determinants recognized by rheumatoid arthritis-specific autoantibodies. Journal of $\mathrm{Cl}$ inical Investigation, 101, 273-281.doi:10.1172/JCI1316

[4] Schellekens, G.A., et al.(2000) The diagnostic properties of rheumatoid arthritis antibodies recognizing a cyclic citrullinated peptide. Arthritis and Rheumatism, 43, 155-163.

doi:10.1002/1529-0131(200001)43:1<155::AID-ANR20 $\geq 3.0 . \mathrm{CO} ; 2-3$

[5] Nielen, M.M., et al. (2005) Antibodies to citrullinated human fibrinogen (ACF) have diagnostic and prognostic value in early arthritis. Annals of the Rheumatic Diseases, 64, 1199-1204. doi:10.1136/ard.2004.029389

[6] Rantapaa-Dahlqvist, S., et al. (2003) Antibodies against cyclic citrullinated peptide and IgA rheumatoid factor predict the development of rheumatoid arthritis. Arthritis and Rheumatism, 48, 2741-2749. doi:10.1002/art.11223

[7] Cambridge, G., et al. (2003) Serologic changes following B lymphocyte depletion therapy for rheumatoid arthritis. Arthritis and Rheumatism, 48, 2146-2154. doi:10.1002/art.11181
[8] Alessandri, C., et al. (2004) Decrease of anti-cyclic citrullinated peptide antibodies and rheumatoid factor following anti-TNFalpha therapy (infliximab) in rheumatoid arthritis is associated with clinical improvement. Annals of the Rheumatic Diseases, 63, 1218-1221. doi:10.1136/ard.2003.014647

[9] Atzeni, F., et al. (2006) Adalimumab clinical efficacy is associated with rheumatoid factor and anti-cyclic citrullinated peptide antibody titer reduction: A one-year prospective study. Arthritis Research and Therapy, 8, R3. doi:10.1186/ar1851

[10] Chen, H.A., et al. (2006) The effect of etanercept on anti-cyclic citrullinated peptide antibodies and rheumatoid factor in patients with rheumatoid arthritis. Annals of the Rheumatic Diseases, 65, 35-39. doi:10.1136/ard.2005.038851

[11] Vis, M., et al. (2008) IgM-Rheumatoid factor, anti-cyclic citrullinated peptide, and anti-citrullinated human fibrinogen antibodies decrease during treatment with the tumor necrosis factor blocker infliximab in patients with rheumatoid arthritis. Journal of Rheumatology, 35, 425-428.

[12] Van Tubergen, A., et al. (2001) Combined spa-exercise therapy is effective in patients with ankylosing spondylitis: A randomized controlled trial. Arthritis and Rheumatism, 45, 430-438. doi:10.1002/1529-0131(200110)45:5<430::AID-ART362 $>3.0 . \mathrm{CO} ; 2-\mathrm{F}$

[13] Berglin, E., et al. (2006) Radiological outcome in rheumatoid arthritis is predicted by presence of antibodies against cyclic citrullinated peptide before and at disease onset, and by IgA-RF at disease onset. Annals of the Rheumatic Diseases, 65, 453-458. doi:10.1136/ard.2005.041376

[14] Agrawal, S., Misra, R. and Aggarwal, A. (2007) Autoantibodies in rheumatoid arthritis: Association with severity of disease in established RA. Clinical Rheumatology, 26, 201-204. doi:10.1007/s10067-006-0275-5

\section{ABBREVIATIONS}

Anti-CCP, antibodies against cyclic citrullinated proteins; AS, Ankylosing sponylitis; BASDAI, Bath ankylosing spondylitis disease activity index; BASFI, Bath ankylos- ing spondylitis functional index; BASMI, bath ankylosing spondylitis metrology index; Ig, Immunoglobuline; LDRnHT, low-dose radon and hyperthermia treatment; NSAID, non-steroid anti-inflammatory drug; RA, Rheumatoid arthritis; TNF- $\alpha$, Tumor necrosis factor $\alpha$. 